\title{
A checklist and areography of Buprestidae, Vesperidae and Cerambycidae (Coleoptera) in Abruzzo, Lazio and Molise National Park, Italy
}

\author{
Vladimir Sakalian, Enrico Migliaccio² ${ }^{2}$ Franco Tassi ${ }^{3}$, Georgi Georgiev $^{4}$
}

1 Institute of Biodiversity and Ecosystem Research, Bulgarian Academy of Sciences, 1 Tzar Osvoboditel Blvd., 1000 Sofia, Bulgaria

2 Science Naturali ed Ambientali, 47 Via Duccio Galimberti, 00136 Rome, Italy

3 Centro Parchi Internazionale, Villino16/4 - 330 Via le Gorgia di Leontini, 00124 Rome, Italy

4 Forest Research Institute, Bulgarian Academy of Sciences, 132 St. Kliment Ohridski Blvd., 1756

Sofia, Bulgaria

Corresponding author: Georgi Georgiev (ggeorgiev.fri@gmail.com)

Received 30 July 2021 | Accepted 2 November 2021 | Published 31 December 2021

Citation: Sakalian V, Migliaccio E, Tassi F, Georgiev G (2021) A checklist and areography of Buprestidae, Vesperidae and Cerambycidae (Coleoptera) in Abruzzo, Lazio and Molise National Park, Italy. Travaux du Muséum National d’Histoire Naturelle “Grigore Antipa” 64(2): 35-60. https://doi.org/10.3897/travaux.64.e72145

\begin{abstract}
The species of Buprestidae, Vesperidae and Cerambycidae in Abruzzo, Lazio and Molise National Park in Italy were studied based on literature data and original material. As a result, a list of 128 taxa was assembled: 49 buprestids, one vesperid and 78 cerambycids. These taxa belong to 21 areographical categories combined in seven complexes. The European complex occupies a dominant position (39.1\%), followed by the Palaearctic (24.2\%), Mediterranean (14.1\%), Eurosiberian (10.2\%), EuropeanIranoturanian (6.3\%), Endemic (3.9\%), and Cosmopolitan and Holarctic (2.3\%) complexes. The Park has four endemic and one subendemic taxa.
\end{abstract}

\section{Keywords}

Abruzzo, Lazio and Molise National Park, endemics, jewel beetles, longhorn beetles. 


\section{Introduction}

Since 1922, the National Park Abruzo, Lazio and Molise has been founded as a protected area under the name Abruzzo N.P. It is the oldest National Park in Italy located exactly in the centre of the Italian Peninsula, a particularly rich landscape, whose historical, geographical, climatic and environmental complexity is perhaps unique in Earth history. The Park's area is about $500 \mathrm{~km}^{2}$, surrounded by a buffer zone of $800 \mathrm{~km}^{2}$. The greatest part of this territory is mountainous (the highest peak is 2,249 $\mathrm{m}$ a.s.l.). Specific relief, oreographic patterns as well as the large valleys of the rivers Sangro, Giovenco and Melfa provide habitats for a great variety of natural flora and fauna. The predominant tree species in the Park is the common beech (Fagus sylvatica) which covers most of the area, generally between 900 and $1800 \mathrm{~m}$ a.s.l. At lower altitudes, some oak species (Quercus spp.) are distributed. The Austrian pine (Pinus nigra), dwarf mountain pine (Pinus mugo) and silver birch (Betula pendula) are other notable trees of the Park. The highest regions are covered by grasslands.

The first entomological research, carried on in Abruzzo before the foundation of the National Park (1922), goes back to Raffray (1914) on Pselaphidae (Coleoptera). A year later, Festa (1915) published the outcome of a research on different insect orders of the upper Sangro valley. Later, Luigioni $(1931,1933)$ started in-depth studies on beetles and some other insect orders. Since then, many other entomological studies have been conducted: e.g., the researches of Cerruti (1940), particularly on Staphylinidae (Coleoptera); the study on Diptera (Saccà and Rivosecchi 1958); the investigations on Plecoptera and Odonata (Consiglio 1952, 1955a, 1955b). Grandi (1958) published the results of an entomological expedition in the National Park during which insects from different orders were collected. In 1957, a group of Roman entomologists (Giovani Papini, Enrico Migliaccio and Franco Tassi) started a systematic investigation of the entomofauna, in cooperation with the Park Agency. In 1993, the Abruzzo National Park started the Biodiversity Project, with the aim to make an inventory of all the species living in the Park. Two checklists regarding Coleoptera have been published so far: Vigna Taglianti (1995) on Carabidae and Carpaneto et al. (1996) on Lamellicornia. In addition, Vigna Taglianti (1994) describes a new species of ground beetle, Ocys tassii. Recently, new taxa and distributional data of endemic and threatened buprestid and cerambycid species in Abruzo, Lazio and Molise National Park have been reported (Sakalian et al. 2020). New papers on the ecology, ethology and distribution of xylophagous beetles are in preparation by Franco Tassi and colleagues.

The aim of this study is to summarise the published data on the jewel and longhorn beetles in Abruzo, Lazio and Molise National Park, to report additionally collected material and published records not mentioned in Sakalian et al. (2020) and to make a zoogeographical analysis of the buprestid and cerambycid fauna in the National Park. 


\section{Material and methods}

Since 1957, Franco Tassi and Enrico Migliaccio have collected many different coleopteran species in Abruzo, Lazio and Molise National Park including, several jewel and longhorn beetles. During the collecting trips of Bulgarian entomologists in the National Park (Mario Langourov and Stoyan Beshkov in 2001 and Toshko Ljubomirov in 2011), some buprestids and cerambycids were also found.

The following traditional methods were used for collecting: hand collecting on flowers and food plants; collecting using the entomological net on grasses and bushes; shaking of tree branches and crowns and collecting the fallen insects; collecting with Malaise traps.

The taxonomic arrangement and zoogeographical characterization were made on the basis of system and recent taxa distribution of buprestids (Kubán et al. 2016) and cerambycids (Danilevsky 2021). According to Sakalian (1994), Georgiev and Hubenov (2006) and Sakalian and Langourov (2007) conceptions, the established taxa are arranged in 21 chorotypes (areographic categories).

The collected specimens were deposited in the following collections: Enrico Migliaccio (EM); Franco Tassi (FT); Institute of Biodiversity and Ecosistem Research, Bulgarian Academy of Sciences (IBER); Istituto Nazionale Entomologia (Italy) (INE); Umberto Pessolano (UP); Pierpaolo Rapuzzi (PR).

\section{Results}

Overall, 128 taxa from three families were identified so far in Abruzzo, Lazio and Molise National Park. (49 Buprestidae, one Vesperidae and 78 Cerambycidae).

Family Buprestidae Leach, 1815

Subfamily Polycestinae Lacordaire, 1857

Tribe Acmaeoderini Kerremans, 1893

Acmaeodera (Acmaeodera) pilosellae pilosellae (Bonelli, 1812)

Colli Nascosi (Luigioni 1933); Scanno, 1000 m a.s.l., 29.06.1996, 1 ex., leg. E. Migliaccio (EM).

Chorotype: Transmediterranean.

Acmaeodera (Palaeotethya) bipunctata bipunctata (Olivier, 1790)

Roccaraso (Gobbi 1970).

Chorotype: Northeast Mediterranean. 
Acmaeoderella (Acmaeoderella) discoida (Fabricius, 1787)

Val Fondillo (Curletti 1994).

Chorotype: Southwest Mediterranean.

Acmaeoderella (Acmaeoderella) circassica Reitter, 1890

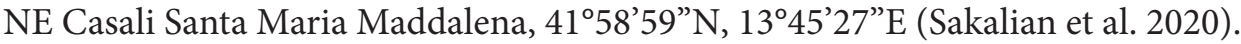

Chorotype: Pontomediterranean.

Acmaeoderella (Carininota) flavofasciata flavofasciata (Piller and Mitterpacher 1783)

Val Fondillo, colle dell'oro (Luigioni 1933); Pratorosso, Pescasseroli, M. Tranquillo, Colle Licco; rive del Sangro, Val Resione, Vallone Pesco di Lordo (Tassi 1962a); Pescasseroli, July 1957, 1 ex., leg. S. Cafaro; Pratorosso, 1536 m a.s.l., 20.07.1995, 1 ex., leg. E. Migliaccio (EM); La Camosciara, $41^{\circ} 46^{\prime} 35^{\prime \prime N}$, $13^{\circ} 54^{\prime} 28^{\prime \prime} \mathrm{E}, 1120 \mathrm{~m}$ a.s.l.,

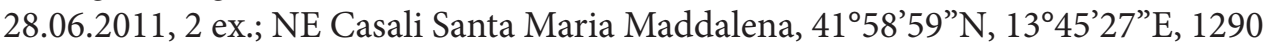

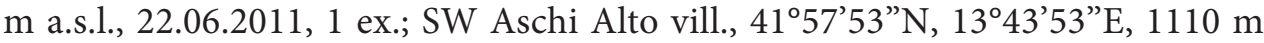
a.s.l., 30.06.2011, 1 ex.; S Ortona dei Marsi vill., 41 ${ }^{\circ} 59^{\prime} 34^{\prime \prime} \mathrm{N}, 13^{\circ} 44^{\prime} 01^{\prime \prime} \mathrm{E} 910 \mathrm{~m}$ a.s.l., 23.06.2011, 2 ex., leg. T. Ljubomirov (IBER).

Chorotype: Southwest Palaearctic.

Tribe Ptosimini Kerremans, 1902

Ptosima undecimmaculata undecimmaculata (Herbst, 1784)

Sperone (Sakalian et al. 2020).

Chorotype: Euromediterranean.

Subfamily Chrysochroinae Laporte, 1835

Tribe Dicercini Gistel, 1848

Capnodis cariosa cariosa (Pallas, 1776)

San Sebastiano dei Marsi (Sakalian et al. 2020).

Chorotype: Pontomediterranean.

Capnodis tenebricosa tenebricosa (Olivier, 1790)

S. Sebastiano dei Marsi (Curletti 1994); Ortona dei Marsi, 1000 m a.s.l., July 2011, 2 ex., leg. E. Migliaccio (EM).

Chorotype: Southwest Palaearctic.

Capnodis tenebrionis (Linnaeus, 1760)

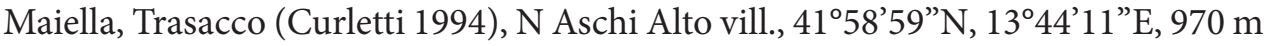
a.s.l., 23.06.2011, 2 ex., leg. T. Ljubomirov (IBER).

Chorotype: Southwest Palaearctic. 
Dicerca (Hemidicerca) aenea aenea (Linnaeus, 1760)

National Park (Curletti 1994).

Chorotype: West Palaearctic.

Dicerca (Hemidicerca) alni (Fischer, 1824)

Pratorosso, M. Tranquillo, Vallone Pesco di Lordo (Tassi 1962a).

Chorotype: Euromediterranean.

Subfamily Buprestinae Leach, 1815

Tribe Anthaxiini Gory and Laporte, 1837

Anthaxia (Anthaxia) chevrieri Gory and Laporte, 1839

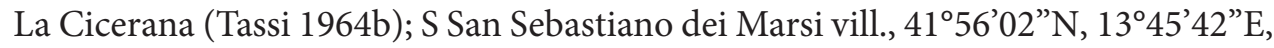

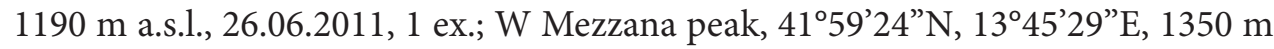

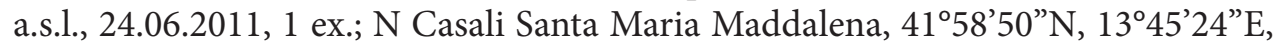
$1220 \mathrm{~m}$ a.s.l., $22.06 .2011,1$ ex.; $41^{\circ} 59^{\prime} 07^{\prime \prime} \mathrm{N}, 13^{\circ} 45^{\prime} 05^{\prime \prime} \mathrm{E}, 1080 \mathrm{~m}$ a.s.l., $24.06 .2011,5$ ex., leg. T. Ljubomirov (IBER).

Chorotype: European.

Anthaxia (Anthaxia) fulgurans (Schrank, 1789)

Val Camosciara (Tassi 1964b).

Chorotype: European.

Anthaxia (Anthaxia) hackeri Frivaldszky, 1884

Fucino (Tassi 1980); Cerchio (Curletti 1994)

Chorotype: Easteuropean-Anatolian.

Anthaxia (Anthaxia) lucens Küster, 1852

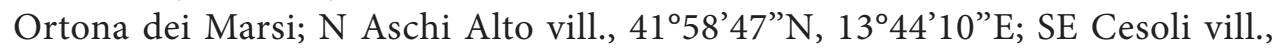
$42^{\circ} 00^{\prime} 36^{\prime \prime} \mathrm{N}, 13^{\circ} 43^{\prime} 32^{\prime \prime} \mathrm{E}$ (Sakalian et al. 2020).

Chorotype: Northeast Mediterranen.

Anthaxia (Anthaxia) nitidula (Linnaeus, 1758)

M. Palombo (Luigioni 1933); Colli Nascosi (Tassi 1962a); Feudo Intramonti Reserve

- Casone Antonucci Place, near Sambuco Spring, 1150 m a.s.l., 06-26.06.2001; 26.06-27.07.2001, 2 ex., leg. P. Tollis (Malaise trap) (EM); SW Aschi Alto vill., $41^{\circ} 57^{\prime} 53^{\prime \prime} \mathrm{N}, 13^{\circ} 43^{\prime} 53^{\prime \prime} \mathrm{E}, 1110 \mathrm{~m}$ a.s.1., $30.06 .2011,1$ ex.; N Villetta Barrèa, $41^{\circ} 48^{\prime} 15^{\prime \prime} \mathrm{N}$, $13^{\circ} 56^{\prime} 09^{\prime \prime}$ E, 1390 m a.s.l., 25.06.2011, 1 ex., leg. E. Migliaccio; SE Casali Santa Maria Maddalena, $41^{\circ} 59^{\prime} 19^{\prime \prime} \mathrm{N}, 13^{\circ} 44^{\prime} 18^{\prime \prime} \mathrm{E}, 910 \mathrm{~m}$ a.s.l., 24.06.2011, 1 ex.; S Cesoli vill., $42^{\circ} 00^{\prime} 19^{\prime \prime} \mathrm{N}, 13^{\circ} 43^{\prime} 08^{\prime \prime} \mathrm{E}, 850 \mathrm{~m}$ a.s.l., 27.06.2011, 2 ex., leg. T. Ljubomirov (IBER).

Chorotype: European. 


\section{Anthaxia (Anthaxia) semicuprea Küster, 1851}

Val Camosciara (Tassi 1962a); Val Fondillo (Curletti 1994); Valle dell'inferno, Pescasseroli, Pescocostanzo (Curletti 1994); Pescocostanzo: bosco S. Antonio, 03.08.1990, 1 ex., leg. E. Migliaccio; Feudo Intramonti, 26.06-27.07.2001, 1 ex., leg. M. Langourov; Feudo Intramonti Reserve - Casone Antonucci Place, near Sambuco Spring, 1150 m a.s.l., 06-26.06.2001; 26.06-27.07.2001, 2 ex., leg. P. Tollis (Malaise trap) (EM); S Bisegna vill., 41 ${ }^{\circ} 53^{\prime} 03^{\prime \prime N}, 13^{\circ} 45^{\prime} 44^{\prime \prime} \mathrm{E}, 1310 \mathrm{~m}$ a.s.l., 19.06.2011, 1 ex.; S Ortona dei Marsi vill., 41 ${ }^{\circ} 59^{\prime} 34^{\prime \prime} \mathrm{N}, 1^{\circ} 44^{\prime} 01^{\prime \prime} \mathrm{E}, 910 \mathrm{~m}$ a.s.l., 21.06.2011, 1ex.; S

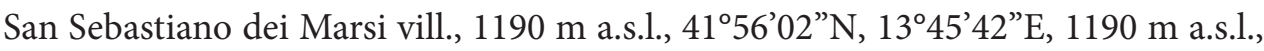
26.06.2011, 1 ex., leg. T. Ljubomirov (IBER).

Chorotype: European-Anatolian.

Anthaxia (Anthaxia) thalassophila thalassophila Abeille de Perrin, 1900

Val Fondillo (Tassi 1962a); Val Fondillo; Scanno (Curletti 1994); Picinisco, June 1968, 2 ex., leg. Eitschberger (EM); S Introdaqua vill., 4200'03"N, 1354'03"E, 630

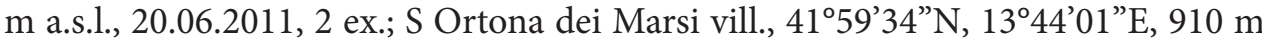
a.s.l., 21.06.2011, 1 ex.; NE Casali Santa Maria Maddalena, 41 ${ }^{\circ} 58^{\prime} 59^{\prime \prime} \mathrm{N}, 13^{\circ} 45^{\prime} 27^{\prime \prime} \mathrm{E}$,

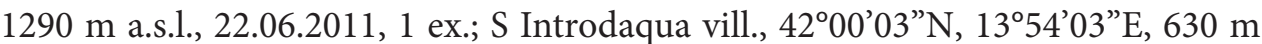
a.s.l., 20.06.2011, 2 ex.; S Cesoli vill., 42 $2^{\circ} 00^{\prime} 19^{\prime \prime} \mathrm{N}, 13^{\circ} 43^{\prime} 08^{\prime \prime} \mathrm{E}, 850 \mathrm{~m}$ a.s.l., 27.06.2011, 12 ex., leg. T. Ljubomirov (IBER).

Chorotype: Northmiddle Mediterranean.

Anthaxia (Cratomerus) hungarica hungarica (Scopoli, 1772)

Pizzone; Scanno: Frattura; NE Civitella peak, $41^{\circ} 59^{\prime} 25^{\prime \prime} \mathrm{N}, 13^{\circ} 43^{\prime} 03^{\prime \prime} \mathrm{E}$; S Introdaqua vill., $42^{\circ} 00^{\prime} 03^{\prime \prime} \mathrm{N}, 13^{\circ} 54^{\prime} 03^{\prime \prime} \mathrm{E}$; S Cesoli vill., $42^{\circ} 00^{\prime} 19^{\prime \prime} \mathrm{N}, 13^{\circ} 43^{\prime} 08^{\prime \prime} \mathrm{E}$ (Sakalian et al. 2020).

Chorotype: Euromediterranean.

Anthaxia (Haplanthaxia) scylla Levey, 1985

Scanno (Sakalian et al. 2020).

Chorotype: Subendemic (Italy and Malta).

Anthaxia (Haplanthaxia) cichorii (Olivier, 1790)

N Castel di Sangro, $41^{\circ} 48^{\prime} 14^{\prime \prime} N, 14^{\circ} 06^{\prime} 23^{\prime \prime} E$; S Ortona dei Marsi vill., 41 $59^{\circ} 34^{\prime \prime} \mathrm{N}$,

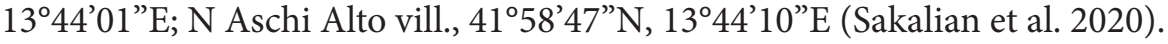

Chorotype: European-Iranoturanian.

Anthaxia (Haplanthaxia) millefolii polychloros Abeille de Perrin, 1894

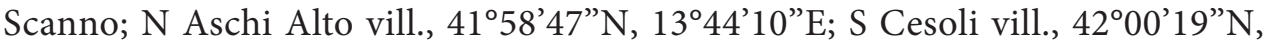
$13^{\circ} 43^{\prime} 08^{\prime \prime}$ (Sakalian et al. 2020).

Chorotype: West European. 
Anthaxia (Melanthaxia) godeti Gory and Laporte, 1839

M. Mattone, Balzo della Chiesa (Tassi 1964a); Val Camosciara, Villetta Barrea (Curletti 1994).

Chorotype: Euromediterranean.

Anthaxia (Melanthaxia) helvetica appenina Obenberger, 1938

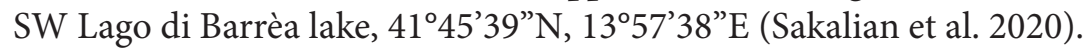

Chorotype: Endemic.

Anthaxia (Melanthaxia) istriana Rosenhauer, 1847

Macchiarvana (Tassi 1962a); Roccaraso (Curletti 1994); Valle Fondillo, 4145'32”N, 135'3'ㄹ, 1140 m a.s.l., 18.06.2011, 1 ex., leg. T. Ljubomirov (IBER).

Chorotype: European-Anatolian.

Anthaxia (Melanthaxia) liae Gobbi, 1983

Scanno; La Camosciara, $41^{\circ} 46^{\prime} 35^{\prime \prime} \mathrm{N}, 13^{\circ} 54^{\prime} 28^{\prime \prime} \mathrm{E}$; S Introdaqua vill., $42^{\circ} 00^{\prime} 03^{\prime \prime} \mathrm{N}$,

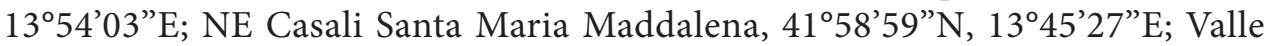
Fondillo, $41^{\circ} 45^{\prime} 10^{\prime \prime} \mathrm{N}, 13^{\circ} 52^{\prime} 00^{\prime \prime} \mathrm{E}$; S Introdaqua vill., $42^{\circ} 00^{\prime} 03^{\prime \prime} \mathrm{N}, 13^{\circ} 54^{\prime} 03^{\prime \prime} \mathrm{E}$; Civitella Alfedena, $41^{\circ} 46^{\prime} 04^{\prime \prime} \mathrm{N}, 13^{\circ} 56^{\prime} 40^{\prime \prime} \mathrm{E}$; N Casali Santa Maria Maddalena, $41^{\circ} 59^{\prime} 07^{\prime \prime} \mathrm{N}$, $13^{\circ} 45^{\prime} 05^{\prime \prime} \mathrm{E}$ (Sakalian et al. 2020).

Chorotype: Endemic.

Tribe Buprestini Leach, 1815

Buprestis (Ancylocheira) cupressi Germar 1836

Maiella (Tassi 1980); Valle del Sangro (Curletti 1994).

Chorotype: East Mediterranean.

Buprestis (Ancylocheira) novemmaculata novemmaculata Linnaeus, 1767

Scanno (Sakalian et al. 2020).

Chorotype: Transpalaearctic.

Buprestis (Ancylocheira) rustica rustica Linnaeus, 1758

National Park (Curletti 1994).

Chorotype: Eurosiberian.

Buprestis (Buprestis) octoguttata octoguttata Linnaeus, 1758

Civitella Alfedena (Sakalian et al. 2020).

Chorotype: West Eurosiberian. 
Tribe Chrysobothrini Gory and Laporte, 1938

Chrysobothris (Chrysobothris) affinis affinis (Fabricius, 1794)

Pratorosso, Pescasseroli, M. Tranquillo, M. Panico, Vallone Pesco di Lordo (Tassi 1962a); Val Fondillo, Roccaraso (Curletti 1994); Val Fondillo, 10.07.1957, 1 ex., leg. F. Cassola; Pescasseroli July 1957, 4 ex., leg. S. Cafaro; Val Fondillo, July 1978, 1 ex., leg. E. Migliaccio; Scanno, 29.06.1996, 4 ex., leg. E. Migliaccio; Feudo Intramonti Reserve - Casone Antonucci Place, near Sambuco Spring, 1150 m a.s.l., 06-26.06.2001, 2 ex., leg. P. Tollis (Malaise trap); Vallone Pesco di Lordo, Ciamamaruca Place, 1560 m a.s.l., 26.06-28.07.2001, leg. P. Tollis (Malaise trap) (EM).

Chorotype: European-Iranian.

Chrysobothris (Chrysobothris) solieri Laporte and Gory, 1837

N Aschi Alto vill., $41^{\circ} 58^{\prime} 59^{\prime \prime} \mathrm{N}, 13^{\circ} 44^{\prime} 11^{\prime \prime} \mathrm{E}$ (Sakalian et al. 2020).

Chorotype: Sothwest Palaearctic.

Tribe Melanophilini Bedel, 1921

Melanophila acuminata (De Geer, 1774)

Pescasseroli (Sakalian et al. 2020).

Chorotype: Transholarctic.

Phaenops cyanea (Fabricius, 1775)

Scanno (Sakalian et al. 2020).

Chorotype: Transpalaearctic.

Subfamily Agrilinae Laporte, 1835

Tribe Agrilini Laporte, 1835

\section{Agrilus angustulus angustulus (Illiger, 1803)}

Prato della Corte, M. Palombo (Luigioni 1933); Pescocostanzo (Curletti 1994); Feudo Intramonti, 1150 m a.s.l., 26.06-27.07.2001, 2 ex., leg. M. Langourov; Feudo Intramonti Reserve - Casone Antonucci Place, near Sangro River, 1000 m a.s.l., 26.06-27.07.2001, leg. P. Tollis (Malaise trap); Feudo Intramonti Reserve - Casone Antonucci Place, near Sambuco Spring, 1150 m a.s.l., 06-26.06.2001, 2 ex.; 26.06-27.07.2001, 3 ex., leg. P. Tollis (Malaise trap) (EM); N Casali Santa Maria Maddalena, 41 $599^{\prime} 07^{\prime \prime} \mathrm{N}, 13^{\circ} 45^{\prime} 05^{\prime \prime} \mathrm{E}$, 1080 m a.s.l., 24.06.2011, 1 ex., leg. T. Ljubomirov (IBER).

Chorotype: Euromediterranean. 
Agrilus biguttatus (Fabricius, 1777)

Assergi, Collemeluccio (Curletti 1994); Feudo Intramonti Reserve - Casone Antonucci Place, near Sambuco Spring, 1150 m a.s.l., 06-26.06.2001, leg. P. Tollis (Malaise trap) (EM).

Chorotype: Euromediterranean.

Agrilus cuprescens cuprescens Ménétriés, 1832

Lago di Barrea (Tassi 1962a); Cerchio (Curletti 1994); Feudo Intramonti Reserve

- Casone Antonucci Place, near Sangro River, 1000 m a.s.l., 06-26.06.2001, leg. P.

Tollis (Malaise trap); Vallone Pesco di Lordo, Ciamamaruca Place, 1560 m a.s.l., 28.07-21.08.2001, leg. M. Langourov and S. Beshkov (EM).

Chorotype: Transpalaearctic.

\section{Agrilus cyanescens cyanescens Ratzeburg, 1837}

Villetta Barrea (Curletti 1994); Feudo Intramonti Reserve - Casone Antonucci Place, near Sambuco Spring, 1150 m a.s.l., 26.06-27.07.2001, leg. P. Tollis (Malaise trap) (EM).

Chorotype: Transpalaearctic.

\section{Agrilus graminis Kiesenwetter, 1857}

Pescocostanzo (Tassi 1962b); National Park (Curletti 1994).

Chorotype: Euromediterranean.

Agrilus hyperici (Creutzer, 1799)

Cerchio (Curletti 1994).

Chorotype: Euromediterranean.

Agrilus obscuricollis Kiesenwetter, 1857

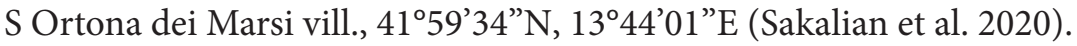

Chorotype: Southwest Palaearctic.

Agrilus olvicolor Kiesenwetter, 1857

SE Cesoli vill., 4200'36”N, 13²3’32”E, 920 m a.s.l., 27.06.2011, 5 ex., leg. T. Ljubomirov (IBER).

Chorotype: European.

\section{Agrilus sulcicollis Lacordaire, 1835}

Pescasseroli (Luigioni 1931); S Rocca Pia vill., 41 ${ }^{\circ} 54^{\prime} 51^{\prime \prime} \mathrm{N}, 13^{\circ} 58^{\prime} 56^{\prime \prime} \mathrm{E}, 1290 \mathrm{~m}$ a.s.l.,

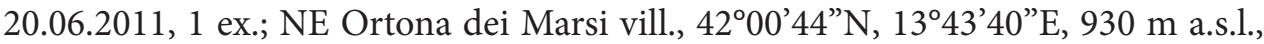
26.06.2011, 1 ex., leg. T. Ljubomirov (IBER).

Chorotype: West Palaearctic. 
Agrilus suvorovi Obenberger, 1935

National Park (Luigioni 1931); Pescasseroli (Curletti 1994); Scanno: Passo Godi, 1450 m a.s.l., 30.06.1996, 1 ex., leg. E. Migliaccio (EM).

Chorotype: Transpalaearctic.

Agrilus viridicaerulans rubi Schaefer, 1937

Castel di Sangro (Tassi 1966); Forca d'Acero (Curletti 1994).

Chorotype: Euromediterranean.

\section{Agrilus viridis (Linnaeus, 1758)}

M. La Rocca, M.Palombo, Terraegna (Luigioni 1933); Pratorosso, Colli Nascosi, Val Fondillo (Tassi 1962a); Pescocostanzo, Roccaraso (Curletti 1994); Feudo Intramonti Reserve - Casone Antonucci Place, near Sangro River, 1000 m a.s.l., 26.06-27.07.2001, leg. P. Tollis (Malaise trap) (EM).

Chorotype: Transpalaearctic.

\section{Tribe Coraebini Bedel, 1921}

\section{Coroebus elatus (Fabricius, 1787)}

M. Mattone (Tassi 1964b); Rocca di mezzo, Scanno (Curletti 1994); Vallone Pesco di Lordo, 1560 m a.s.l., 28.07-21.08.2002, 1 ex., leg. M. Langourov; Feudo Intramonti Reserve - Casone Antonucci Place, near Sangro River, 1000 m a.s.l., 26.06-27.07.2001, 2 ex., leg. P. Tollis (Malaise trap); Feudo Intramonti Reserve - Casone Antonucci Place, near Sambuco Spring, 1150 m a.s.l., 26.06-27.07.2001, 4 ex., leg. P. Tollis (Malaise trap); 27.07-20.08.2001, 5 ex., leg. M. Langourov and S. Beshkov (EM); N Villetta Barrèa, $41^{\circ} 48^{\prime} 15^{\prime \prime} \mathrm{N}, 13^{\circ} 56^{\prime} 09^{\prime \prime} \mathrm{E}, 1390$ m a.s.l., 25.06.2011, 2 ex.; La Camosciara,

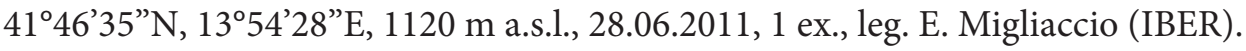

Chorotype: West Palaearctic.

\section{Coroebus rubi (Linnaeus, 1767)}

Cerchio, Pescasseroli (Curletti 1994).

Chorotype: Euromediterranean.

Tribe Tracheini Laporte, 1835

Trachys minutus minutus (Linnaeus, 1758)

Val Fondillo, Prato della Corte (Luigioni 1933); M. Panico (Tassi 1962a).

Chorotype: Transpalaearctic. 
Family Vesperidae Mulsant, 1839

Subfamily Vesperinae Mulsant, 1839

Tribe Vesperini Mulsant, 1839

Vesperus luridus (Rossi, 1794)

Monte Sirente (Sama 1988).

Chorotype: North Mediterranean.

Family Cerambycidae Latreille, 1802

Subfamily Prioninae Latreille, 1802

Tribe Prionini Latreille, 1802

Prionus coriarius (Linnaeus, 1758)

Roccaraso; La Cicerana (Sakalian et al. 2020).

Chorotype: West Palaearctic.

Tribe Aegosomatini J. Thomson, 1861

Aegosoma scabricorne (Scopoli, 1763)

Pescasseroli; Opi; Ortona dei Marsi (Sakalian et al. 2020).

Chorotype: European-Iranian.

Subfamily Lepturinae Latreille, 1802

Tribe Lepturini Latreille, 1802

Grammoptera ruficornis ruficornis (Fabricius, 1781)

Villetta Barrea (Parenti 1964).

Chorotype: European-Anatolian.

Leptura (Leptura) aurulenta Fabricius, 1793

Monte Tranquillo, Val Fondillo (Luigioni 1931); Pratorosso, 10.08.1960, 1 ex., leg. A. Parenti (UP); Pratorosso; Monte Tranquillo (Parenti 1962); Rif. Pratorosso; Monte Tranquillo (Sama 1988); Val Fondillo, 18.07.1995, 1 ex., leg. E. Migliaccio (EM).

Chorotype: Euromediterranean. 
Pseudovadonia livida livida (Fabricius, 1776)

Fonte Puzza; Macchia Arvana (Luigioni 1933); Bosco S. Antonio (Tassi 1961); Val Camosciara; Serrone; Rive Fiume Sangro (Parenti 1962); Pescasseroli; Monte Morrone (Sama 1988).

Chorotype: European.

Stenurella (Priscostenurella) bifasciata bifasciata (O. F. Müller, 1776)

Pescasseroli (Luigioni 1931); Serrone (Parenti 1962); Scanno (Sama 1988).

Chorotype: West Eurosiberian.

Stictoleptura (Melanoleptura) scutellata scutellata (Fabricius, 1781)

Pescasseroli (Luigioni 1931); Opi (Sama 1988).

Chorotype: European.

Stictoleptura (Paracorymbia) fulva (De Geer, 1775)

Pescasseroli (Luigioni 1931); Camosciara (Tassi 1961); Serrone (Parenti 1962); Monte Gloria d'Orlando; Barrea (Sama 1988).

Chorotype: European-Anatolian.

Stictoleptura (Stictoleptura) cordigera cordigera (Fuessly, 1775)

Bosco S. Antonio (Tassi 1961) Roccadimezzo; Maiella; Monte Sirente (Sama 1988).

Chorotype: European-Iranian.

Tribe Oxymirini Danilevsky, 1997

Dinoptera collaris (Linnaeus, 1758)

Val Camosciara (Parenti 1962); Pescasseroli, May 1963, 1 ex., leg. L. Costrini (UP);

Val Cavuto; Monte Greco (Sama 1988).

Chorotype: Eurosiberian.

Rhagium (Megarhagium) mordax (De Geer, 1775)

Monte Marsicano, Val di Corte (Tassi 1961); Val Camosciara; Pratorosso; Bosco Schiappito; Vallone Pesco di Lordo (Parenti 1962); Val Fondillo, 01.06.1964, 1 ex., leg. G. Papini (EM).

Chorotype: Eurosiberian.

Rhagium (Megarhagium) sycophanta (Schrank, 1781)

Roccaraso (Sama 1988).

Chorotype: West Eurosiberian.

Stenocorus (Stenocorus) meridianus (Linnaeus, 1758)

Between Villetta Barrea and fork for Val Fondillo (Parenti 1964).

Chorotype: Eurosiberian. 
Tribe Rhamnusiini Sama, 2009

Rhamnusium bicolor bicolor (Schrank, 1781)

Fonte di Schiappito (Luigioni 1933); Barrea; Cerchio (Sama 1988).

Chorotype: European.

Subfamily Necydalinae Latreille, 1825

Necydalis (Necydalis) ulmi (Chevrolat, 1838)

Cerchioi; Ortona dei Marsi (Sakalian et al. 2020).

Chorotype: European-Iranian.

Subfamily Spondylidinae Audinet-Serville, 1832

Tribe Asemini J. Thomson, 1861

Arhopalus ferus (Mulsant, 1839)

Abruzzo National Park (Grandi 1958); Roccaraso (Parenti 1966); Ortona dei Marsi, 1000 m a.s.l., July-August 2010; September 2012, 4 ex., leg. E. Migliaccio (EM).

Chorotype: Transpalaearctic.

Tribe Saphanini Gistel, 1848

Saphanus piceus piceus (Laicharting, 1784)

Monte Palombo (Luigioni 1931); Monte Tranquillo, Pratorosso (Parenti 1962); Monte Tranquillo, 1500 m a.s.l., 04.07.1960, 1 ex., leg. E. Migliaccio (EM).

Chorotype: European.

Subfamily Cerambycinae Latreille, 1802

Tribe Anaglyptini Lacordaire, 1868

Anaglyptus (Anaglyptus) gibbosus (Fabricius, 1787)

Pescasseroli; Prato di Corte; Barrea; Barrea: Monte Marsicano (Valle Orsara) (Sakalian et al. 2020).

Chorotype: Transmediterranean.

Anaglyptus (Anaglyptus) mysticus (Linnaeus, 1758)

Cesa Crivello; Bosco Schiappito; Bosco Schiappito: Val di Corte Monte Palombo; Abruzzo National Park (Sakalian et al. 2020).

Chorotype: European-Anatolian. 
Tribe Brachypteromatini Sama, 2008

Dolocerus reichii Mulsant, 1862

Pescasseroli (Luigioni 1931).

Chorotype: Northeast Mediterranean.

Tribe Callichromatini Swainson and Shuckard, 1840

Aromia moschata moschata (Linnaeus, 1758)

Abruzzo National Park; Val Camosciara (Sakalian et al. 2020).

Chorotype: Eurosiberian.

Tribe Callidiini Kirby, 1837

Phymatodes (Phymatodes) testaceus (Linnaeus, 1758)

Forca d'Acero: Macchia Arvana; Pescasseroli; Alfedena; Scanno; Ortona dei Marsi (Sakalian et al. 2020).

Chorotype: Transholarctic.

Phymatodes (Poecilium) alni alni (Linnaeus, 1767)

Pescasseroli, Fonte della Difesa (Luigioni 1931); Val Fondillo, 14.05.1994, 1 ex., leg. E. Migliaccio (EM).

Chorotype: European-Anatolian.

Pyrrhidium sanguineum (Linnaeus, 1758)

Pescasseroli; Ortona dei Marsi (Sakalian et al. 2020).

Chorotype: Euromediterranean.

Ropalopus (Ropalopus) clavipes (Fabricius, 1775)

Pescasseroli (Sakalian et al. 2020).

Chorotype: European-Iranian.

Ropalopus (Ropalopus) ungaricus (Herbst, 1784)

Bosco S. Antonio; Alfedena; Roccaraso; Rocca di Mezzo; Rocca di Cambio; Gioia Vecchio; Roccaraso; Passo Godi (Sakalian et al. 2020).

Chorotype: Euromediterranean.

Tribe Cerambycini Latreille, 1802

Cerambyx (Cerambyx) cerdo cerdo Linnaeus, 1758

Caramanico (Sakalian et al. 2020).

Chorotype: Euromediterranean. 
Cerambyx (Microcerambyx) scopolii scopolii Fuessly, 1775

Abruzzo National Park; Vallone Pesco di Lordo; Valle Iannanghera; Valle Schiappito;

Pescasseroli (Sakalian et al. 2020).

Chorotype: European-Anatolian.

\section{Tribe Clytini Mulsant, 1839}

Chlorophorus (Crassofasciatus) trifasciatus (Fabricius, 1781)

La Cicerana (Parenti 1964); Scanno (Sama 1988); Bisegna, 1200 m a.s.l., 24.07.1994;

Ortona dei Marsi, 1000 m a.s.l., June 2012., 2 ex., leg. E. Migliaccio (EM).

Chorotype: Transmediterranean.

Chlorophorus (Humeromaculatus) figuratus (Scopoli, 1763)

Val Fondillo; Valle Camosciara; Roccaraso; Pescasseroli (Sakalian et al. 2020).

Chorotype: West Eurosiberian.

Chlorophorus (Immaculatus) glabromaculatus glabromaculatus (Goeze, 1777)

Pescasseroli; Abruzzo National Park (Sakalian et al. 2020).

Chorotype: European.

Chlorophorus (Immaculatus) varius varius (O. F. Muller, 1766)

Pescasseroli; Val di Corte (Sakalian et al. 2020).

Chorotype: West Eurosiberian.

Chlorophorus (Perderomaculatus) sartor (O. F. Müller, 1766)

Abruzzo National Park; Colle Licco: valley of Sangro; Ortona dei Marsi (Sakalian et al. 2020).

Chorotype: West Palaearctic.

Clytus (Clytus) arietis arietis (Linnaeus, 1758)

Colle della Regina; Campomizzo; Terraegna; Val Fondillo; Valle Iannanghera; Camosciare; Abruzzo National Park; Scanno (Sakalian et al. 2020).

Chorotype: European-Anatolian.

Clytus (Clytus) rhamni rhamni Germar, 1817

Ortona dei Marsi (Sakalian et al. 2020).

Chorotype: Northeast Mediterranean.

Echinocerus floralis (Pallas, 1773)

Abruzzo National Park (Grandi 1958); Serrone (Parenti 1962); Tagliacozzo, July 1990 - July 1991, 4 ex., leg. S. Cafaro; Camosciara, 28.06.2011, 1 ex., leg. E. Migliaccio (EM).

Chorotype: Transpalaearctic. 
Plagionotus arcuatus arcuatus (Linnaeus, 1758)

Pescasseroli; Scanno (Sakalian et al. 2020).

Chorotype: Euromediterranean.

Xylotrechus (Rusticoclytus) rusticus (Linnaeus, 1758)

Schiena Cavallo; Balzo del Caprio; Monte Palombo; Monte La Rocca (Sakalian et al. 2020).

Chorotype: Transpalaearctic.

Xylotrechus (Xylotrechus) arvicola arvicola (Olivier, 1795)

Abruzzo National Park; Piani di Pezza; Rivisondoli (Sakalian et al. 2020).

Chorotype: Euromediterranean.

Tribe Deilini Fairmaire, 1864

Deilus fugax (Olivier, 1790)

Barrea; Pescasseroli; Rivisondoli; Cocullo (Sakalian et al. 2020).

Chorotype: Euromediterranean.

Tribe Graciliini Mulsant, 1839

Axinopalpis gracilis gracilis (Krynicki, 1832)

Cerchio (Sakalian et al. 2020).

Chorotype: European-Iranian.

Gracilia minuta (Fabricius, 1781)

Fontana della Difesa; Cerchio (Sakalian et al. 2020).

Chorotype: Transpalaearctic.

Tribe Rosaliini Fairmaire, 1864

Rosalia (Rosalia) alpina alpina (Linnaeus, 1758)

Pescasseroli; Cesa Crivello; Monte Palombo; Pratorosso; Val Camosciara; Val Fondillo; Val Camosciara; Cicerana (Sakalian et al. 2020).

Chorotype: European-Anatolian.

Tribe Hesperophanini Mulsant, 1839

Hesperophanes sericeus (Fabricius, 1787)

Abruzzo National Park (Sakalian et al. 2020).

Chorotype: Sothwest Palaearctic. 
Trichoferus holosericeus (Rossi, 1790)

Barrea (Parenti 1964); Cerchio; Barrea (Sama 1988); Ortona dei Marsi, 1000 m a.s.l., August 2012, 1 ex., leg. E. Migliaccio (EM).

Chorotype: Euromediterranean.

Tribe Hylotrupini Zagajkevitch, 1991

Hylotrupes bajulus (Linnaeus, 1758)

Abruzzo National Park; Pescasseroli; Castello Favone; Scanno; Lago di Barrea (Sakalian et al. 2020).

Chorotype: Cosmopolitian.

Tribe Molorchini Gistel, 1848

Molorchus (Molorchus) umbellatarus umbellatarus (Schreber, 1759)

Val Camosciara (Parenti 1962); Prato La Corte (Sama 1988).

Chorotype: European-Anatolian.

Tribe Purpuricenini J. Thomson, 1861

Purpuricenus kaehleri kaehleri (Linnaeus, 1758)

Bussi; Pescasseroli (Sakalian et al. 2020).

Chorotype: European-Anatolian.

Tribe Stenopterini Gistel, 1848

Stenopterus rufus rufus (Linnaeus, 1767)

Castello Favone; Barrea; Rocca Tre Monti; Scanno; Cerchio; Lago di Barrea (Sakalian et al. 2020).

Chorotype: European.

Subfamily Lamiinae Latreille, 1825

Tribe Acanthocinini Blanchard, 1845

Acanthocinus (Acanthocinus) xanthoneurus (Mulsant and Rey, 1852)

Monte Palombo; Pescasseroli; Barrea; Val Fondillo (Sakalian et al. 2020).

Chorotype: Endemic.

Leiopus (Leiopus) nebulosus nebulosus (Linnaeus, 1758)

Pescasseroli (Sama 1988); S. Sebastiano dei Marsi, 1200 m a.s.l., 19.06.2011, 1 ex., leg. E. Migliaccio (EM).

Chorotype: European. 
Tribe Agapanthiini Mulsant, 1839

Agapanthia (Agapanthia) cardui (Linnaeus, 1767)

Barrea (Parenti 1964); Monte Greco, Barrea, Passo Godi, Pescasseroli (Sama 1988); Ortona dei Marsi, $900 \mathrm{~m}$ a.s.l., 20-23.06.2009; Roccagorga (Castel di Sangro), $800 \mathrm{~m}$ a.s.l., 20.06.2011; between Carrito and Aschi, 30.06.2011, 3 ex., leg. E. Migliaccio (EM).

Chorotype: European.

Agapanthia (Epoptes) villosoviridescens (DeGeer, 1775)

Abruzzo National Park (Luigioni 1933); Valle fredda, Val Fondillo; Val Resione (Tassi 1961; Parenti 1962); Pescasseroli, Passo Godi, Monte Greco, Monte Marsicano, Gioia Vecchio (Sama 1988).

Chorotype: Eurosiberian.

Agapanthia (Smaragdula) violacea (Fabricius, 1775)

Val Fondillo, Prato della Corte (Luigioni 1933); Abruzzo National Park (Grandi 1958); Fiume Sangro (Parenti 1964); Pescasseroli, Monte Greco, F. Sangro (Sama 1988); Cocullo, 06.05.1993-20.05.1995, 2 ex., leg. S. Cafaro (EM).

Chorotype: European-Anatolian.

Calamobius filum (Rossi, 1790)

Pescasseroli (Parenti 1964); Monte Greco, Gioia Vecchio, Pescasseroli (Sama 1988); Pescasseroli, July 1957, 1 ex., leg. S. Cafaro; Ortona dei Marsi, 900 m a.s.l., 20-23.07.2009, 1 ex. (EM).

Chorotype: West Palaearctic.

Tribe Apodasyini Lacordaire, 1872

Anaesthetis testacea testacea (Fabricius, 1781)

Assergi, Alfedena (Sama 1988).

Chorotype: European-Anatolian.

Tribe Dorcadionini Swainson, 1840

Dorcadion (Cribridorcadion) arenarium marsicanum Fracassi, 1905

Cerchio, Campo di Giove, Monte Tranquillo, Pescasseroli, Forca Caruso, Prato la Corte, La Prata di Lecce (Sama 1988); Val Fondillo, 28.05.1999, 2 ex., leg. E. Migliaccio (EM).

Chorotype: Northmiddle Mediterranean. 
Dorcadion (Cribridorcadion) etruscum etruscum (Rossi, 1790)

Civitella Alfedena; Le Cese; Colle dei gatti; Colli Merini; Lago di Barrea; Cerchio; Ortona dei Marsi (Sakalian et al. 2020).

Chorotype: Endemic following the classification of Danilevsly (2021). Otherwise, a different treatment was proposed by Pesarini and Sabbadini (2007) who considered the species being present in Greece too.

Tribe Lamiini Latreille, 1825

Herophila tristis tristis (Linnaeus, 1767)

Pescasseroli (Luigioni 1931); Valle Iannanghera; Lago di Barrea (Parenti 1964); Ortona dei Marsi, 1000 m a.s.l., July 2010 - July 2012, 8 ex., leg. E. Migliaccio (EM). Chorotype: North Mediterranean.

\section{Lamia textor (Linnaeus, 1758)}

Pescasseroli (Luigioni 1931); Tagliacozzo, Cerchio (Sama 1988); Tagliacozzo, July 1961, 1 ex., leg. G. Montelli (EM).

Chorotype: Transpalaearctic.

\section{Morimus asper asper (Sulzer, 1776)}

Parco d'Abruzzo (Grandi 1958); Pescasseroli, Monte Tranquillo; Vallone Pesco di Lordo; Bosco Schiappito (Parenti 1962); Ortona dei Marsi, October 2010, 1 ex., leg. E. Migliaccio (EM).

Chorotype: North Mediterranean.

Tribe Mesosini Mulsant, 1839

Mesosa (Aplocnemia) nebulosa nebulosa (Fabricius, 1781)

Pescasseroli, Monte Schiena Cavallo, Serra delle Gravare (Luigioni 1931, 1933); Monte Difesa (Sama 1988).

Chorotype: European-Anatolian.

Tribe Phytoeciini Mulsant, 1839

Oberea (Oberea) oculata (Linnaeus, 1758)

Abruzzo National Park (Grandi 1958); Camosciara (Tassi 1961); Val Camosciara, Colle Licco (Parenti 1962); Villetta Barrea (Sama 1988); Val Camosciara, 28.07.1959; 02.08.1960, 5 ex., leg. E. Migliaccio (EM).

Chorotype: Transpalaearctic. 
Phytoecia (Musaria) affinis affinis (Harrer, 1784)

Prato della Corte (Luigioni 1933); Pescasseroli (Parenti 1964); Pescasseroli, Valle di Corte, Gioia Vecchio (Sama 1988).

Chorotype: European-Anatolian.

Phytoecia (Musaria) tirellii (Luigioni, 1913)

Pescasseroli, Monte Sirente (Sama 1988).

Chorotype: Northeast Mediterranean.

Phytoecia (Opsilia) coerulescens (Scopoli, 1763)

Pescasseroli, Val di Corte (Sama 1988); Cocullo, 12.06.1989, 1 ex., leg. S. Cafaro; between Trasacco and Collelongo, 28.05.2005, 1 ex., leg. R. Casalini (EM).

Chorotype: West Palaearctic.

Phytoecia (Opsilia) molybdaena (Dalman, 1817)

Abruzzo National Park (Grandi 1958).

Chorotype: Transpalaearctic.

Phytoecia (Phytoecia) cylindrica (Linnaeus, 1758)

Pescasseroli, Schiena Cavallo (Luigioni 1933); Abruzzo National Park (Grandi 1958); Lago di Barrea (Tassi 1960a); Passo del calvario (Parenti 1962); Pescasseroli, Val di Corte, Forca d'acero (Sama 1988); Abruzzo National Park, 07.1957, 1 ex., leg. S. Cafaro; Lago di Barrea, 25.06.2011; between Cesoli and Carrito, 27.06.2011, 2 ex., leg. E. Migliaccio (EM).

Chorotype: Eurosiberian.

Phytoecia (Phytoecia) nigricornis (Fabricius, 1782)

Pescasseroli, Val Fondillo (Luigioni 1933); Monte Mattone (Parenti 1964; Tassi 1964a); Valle di Corte, S. Sebastiano, Forca d’acero (Sama 1988).

Chorotype: Eurosiberian.

Phytoecia (Phytoecia) pustulata pustulata (Schrank, 1776)

Prato di Corte (Luigioni 1933); Pescasseroli (Sama 1988).

Chorotype: European-Iranoturanian.

Phytoecia (Phytoecia) rufipes rufipes (Olivier, 1795)

Val Fondillo (Sama 1988).

Chorotype: Transpalaerctic.

Phytoecia (Phytoecia) vulneris vulneris Aurivillius, 1923

Cerchio, Monte Greco (Sama 1988).

Chorotype: North Mediterranean. 
Tribe Pogonocherini Mulsant, 1839

Pogonocherus (Pityphilus) fasciculatus fasciculatus (De Geer, 1775)

Pescasseroli (Tassi 1963; Parenti 1964).

Chorotype: Transpalaearctic.

Pogonocherus (Pogonocherus) hispidus (Linnaeus, 1758)

Prato della Corte (Luigioni 1933).

Chorotype: Euromediterranean.

Tribe Saperdini Mulsant, 1839

Saperda (Lopezcolonia) scalaris scalaris (Linnaeus, 1758)

Pescasseroli; Val di Sangro (Sakalian et al. 2020).

Chorotype: Euromediterranean.

Saperda (Saperda) carcharias (Linnaeus, 1758)

Vitelletta Barrea (Tassi 1961); Pescasseroli (Parenti 1962); Pescasseroli, Valle di Corte (Sama 1988).

Chorotype: Transpalaearctic.

Stenostola ferrea ferrea (Schrank, 1776)

Monte Sirente (Sakalian et al. 2020).

Chorotype: European-Anatolian.

Tribe Tetropini Portevin, 1927

Tetrops praeustus praeustus (Linnaeus, 1758)

Villetta Barrea (Sama 1988).

Chorotype: Transpalaearctic.

The identified taxa belong to 21 areographical categories (chorotypes) combined in 7 complexes (Table 1 ).

The European complex occupies a dominant position (39.1\%), followed by the Palaearctic (24.2\%), Mediterranean (14.1\%), Eurosiberian (10.2\%), EuropeanIranoturanian (6.3\%), Endemic (3.9\%) and Cosmopolitan and Holarctic (2.3\%) complexes (Fig. 1). 
Table 1. Areogeographic characterization of buprestid, vesperid and cerambycid species in Abruzzo, Lazio and Molise National Park

\begin{tabular}{|c|c|c|c|c|c|c|}
\hline \multirow{2}{*}{ Areographic categories and complexes } & \multicolumn{2}{|c|}{ Buprestidae } & \multicolumn{2}{|c|}{$\begin{array}{c}\text { Vesperidae, } \\
\text { Cerambycidae }\end{array}$} & \multicolumn{2}{|c|}{ Total } \\
\hline & $\mathbf{N}$ & $\%$ & $\mathbf{N}$ & $\%$ & $\mathbf{N}$ & $\%$ \\
\hline Cosmopolitian and Holarctic complex & 1 & 2.0 & 2 & 2.5 & 3 & 2.3 \\
\hline Cosmopolitan & - & - & 1 & 1.3 & 1 & 0.8 \\
\hline Transholarctic & 1 & 2.0 & 1 & 1.2 & 2 & 1.5 \\
\hline Palaearctic complex & 15 & 30.7 & 16 & 20.3 & 31 & 24.2 \\
\hline Transpalaearctic & 7 & 14.3 & 11 & 13.9 & 18 & 14.1 \\
\hline West Palaearctic & 3 & 6.2 & 4 & 5.1 & 7 & 5.5 \\
\hline Southwest Palaearctic & 5 & 10.2 & 1 & 1.3 & 6 & 4.7 \\
\hline Eurosiberian complex & 2 & 4.0 & 11 & 13.9 & 13 & 10.2 \\
\hline Eurosiberian & 1 & 2.0 & 7 & 8.8 & 8 & 6.3 \\
\hline West Eurosiberian & 1 & 2.0 & 4 & 5.1 & 5 & 3.9 \\
\hline European-Iranoturanian complex & 2 & 4.0 & 6 & 7.6 & 8 & 6.3 \\
\hline European-Iranoturanian & 1 & 2.0 & 1 & 1.3 & 2 & 1.6 \\
\hline European-Iranian & 1 & 2.0 & 5 & 6.3 & 6 & 4.7 \\
\hline European complex & 18 & 34.8 & 32 & 40.5 & 50 & 39.1 \\
\hline Euromediterranean & 10 & 20.4 & 10 & 12.7 & 20 & 15.6 \\
\hline European-Anatolian & 2 & 4.1 & 14 & 17.7 & 16 & 12.5 \\
\hline Easteuropean-Anatolian & 1 & 2.1 & - & - & 1 & 0.8 \\
\hline European & 4 & 8.2 & 8 & 10.1 & 12 & 9.4 \\
\hline West European & 1 & 2.0 & - & - & 1 & 0.8 \\
\hline Mediterranean complex & 8 & 16.3 & 10 & 12.7 & 18 & 14.1 \\
\hline Transmediterranean & 1 & 2.0 & 2 & 2.5 & 3 & 2.3 \\
\hline North Mediterranean & $\begin{array}{c}- \\
-\end{array}$ & - & 4 & 5.1 & 4 & 3.1 \\
\hline Northmiddle Mediterranean & 1 & 2.0 & 1 & 1.3 & 2 & 1.6 \\
\hline East Mediterranean & 1 & 2.0 & - & - & 1 & 0.8 \\
\hline Northeast Mediterranean & 2 & 4.1 & 3 & 3.8 & 5 & 3.9 \\
\hline Southwest Mediterranean & 1 & 2.1 & - & - & 1 & 0.8 \\
\hline Pontomediterranean & 2 & 4.1 & - & - & 2 & 1.6 \\
\hline Endemic complex & 3 & 6.1 & 2 & 2.5 & 5 & 3.9 \\
\hline Italian endemics & 3 & 6.1 & 2 & 2.5 & 5 & 3.9 \\
\hline Total & 49 & 100.0 & 79 & 100.0 & 128 & 100.0 \\
\hline
\end{tabular}




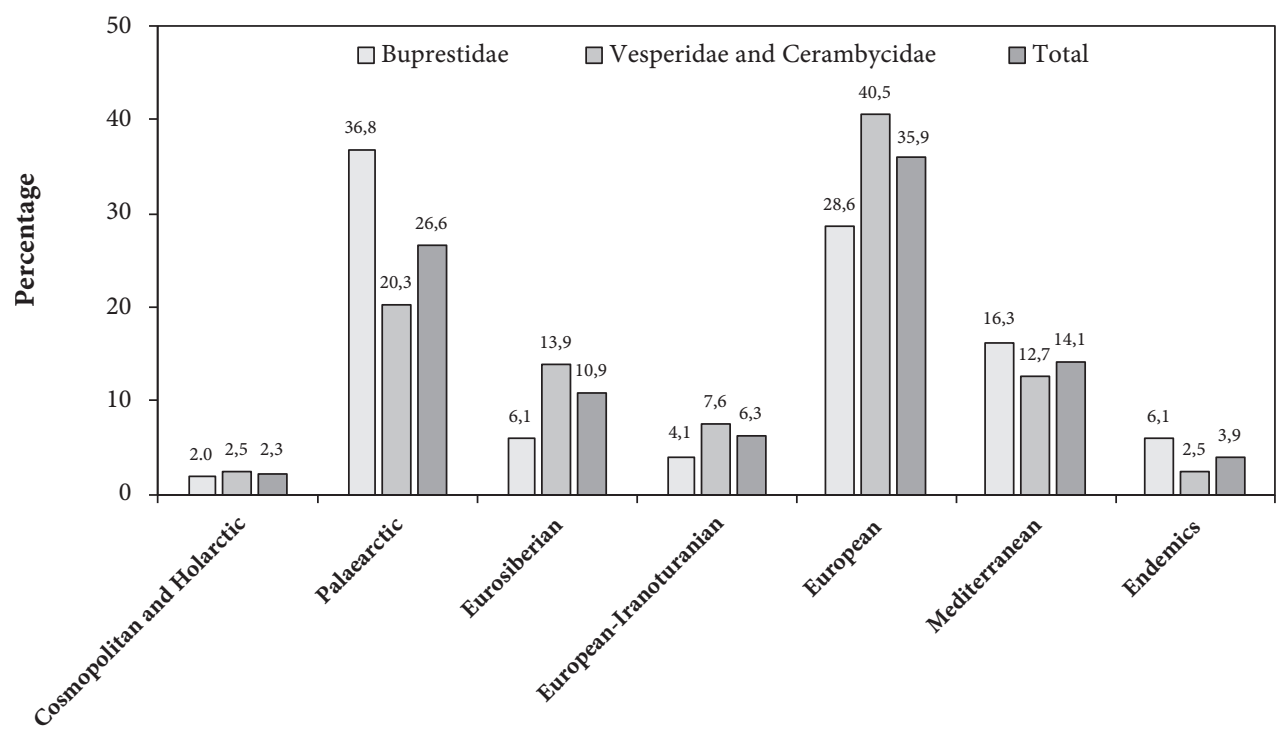

Figure 1. Areographic complexes of buprestid, vesperid and cerambycid species in Abruzzo, Lazio and Molise National Park

\section{Discussion}

Following the study on the species richness and distribution of Buprestidae, Vesperidae and Cerambycidae in the Abruzzo, Lazio and Molise National Park, a total of 128 species and subspecies were found so far but we can expect that future investigations will increase the number of currently known taxa. However, the high biodiversity level, around 24\% of all buprestids known for Italy - 208 (Curletti et al. 2003), around 26\% of all known cerambycids - 296 (Sama and Rapuzzi 2011), and $25 \%$ of the four vesperid taxa (Danilevsky 2021), points to the favorable ecological conditions inside the Park territory for these beetle taxa.

According to the areographic analyses the species and subspecies of the European complex are dominant (39.1\%), and are connected with the deciduous forests which cover the largest part of the National Park territory. This considerable domination is mainly due to the Cerambycidae, with 32 taxa (40.5\%). Secondly, there are species and subspecies belonging to Palaearctic complex (24.2\%). These more euribiont taxa, with broad areas of distribution, normaly are better represented in the high mountains, because of the harsh climatic conditions there. In this complex, on the contrary, buprestids are better represented (30.7\%), followed by cerambycids (20.3\%). Very closely, on the third and fourth position are the taxa belonging to the Medditerranean and Eurosiberian complexes. In the first complex the buprestids are better represented (as a more xerophilic group), and in the second - the cerambycids. In the lower parts and along the rivers climatic conditions allows the penetration of the Mediterranean 
species and subspecies, while the high areas, especially those covered by coniferous trees, are favorable for distribution of the Eurosiberian taxa.

Similar aerographic characteristics concern the Cerambycidae from Vitosha, in Bulgaria, where the European taxa take the first place, followed by the Palaearctic, Eurosiberian and Mediterranean taxa with relatively equal representation (Topalov 2018). Vitosha with its elevation, oreographic patterns and vegetation is similar to Abruzzo, Lazio and Molise National Park.

Abruzzo, Lazio and Molise National Park has four endemic and one subendemic taxa: two Italian endemic buprestids (Anthaxia helvetica apennina and Anthaxia liae), two endemic cerambycids (Acanthocinus xanthoneurus and Dorcadion etruscum etruscum), and one subendemic buprestid (Anthaxia scylla present in Italy and Malta).

In conclusion, it is possible that new taxa from Buprestidae, Vesperidae and Cerambycidae families could be expected in future investigations on the territory of Abruzzo, Lazio and Molise National Park.

\section{Acknowledgements}

We would like to thank Dr. Toshko Ljubomirov (Sofia, Bulgara), Dr. Mario Langourov (Sofia, Bulgara) and Dr. Stoyan Beshkov (Sofia, Bulgara) for giving us the available biological materials for investigation.

\section{References}

Carpaneto G, Migliaccio E, Piattella E (1996) Progetto Biodiversità. Liste preliminari degli organismi viventi nel Parco Nazionale d'Abruzzo (Coleoptera: Lamellicornia) 8: 7-29. [in Italian]

Cerruti M (1940) Prima nota di nuova specie per il Parco Nazionale d’Abruzzo (Coleoptera: Staphylinidae). Bollettino della Società Entomologica Italiana 72: 111. [in Italian]

Consiglio C (1952) Odonati dell'Italia meridionale e degli Abruzzi. Memorie della Società Entomologica Italiana 5(31): 96-108. [in Italian]

Consiglio C (1955a) Due nuove specie di Plecotteri italiani. Bollettino della Società Entomologica Italiana 85(5-6): 70-77. [in Italian]

Consiglio C (1955b) Note sui Plecotteri italiani. Bollettino del Laboratorio di Zoologia Generale e Agraria Filippo Silvestri 33: 273-282. [in Italian]

Curletti G (1994) I Buprestidi d'Italia. Monografie di Natura Bresciana 19, 318 pp. [in Italian] Curletti G, Rastelli M, Rastelli S, Tassi F (2003) Coleotteri Buprestidi d'Italia. Piccole Faune. CD - ROM. ISBN 88-901201-9-3. [in Italian]

Danilevsky ML (2021) A check-list of Longicorn Beetles (Coleoptera, Cerambycoidea) of Europe. Updated 02.03.2021. http://www.cerambycidae.net/catalog.pdf. 
Festa E (1915) Escursioni zoologiche nei monti della vallata del Sangro (Abruzzi) - Parte narrativa. Bollettino dei Musei di Zoologia e di Anatomia comparata della Regia Università di Torino 692(30): 1-27. [in Italian]

Georgiev G, Hubenov Z (2006) Vertical Distribution and Zoogeographical Characteristics of Cerambycidae (Coleoptera) Family in Bulgaria. Acta zoologica bulgarica 58(3): 315-343.

Gobbi G (1970) Contributo alla conoscenza dei Coleotteri Buprestidi d'Italia. Bollettino dell'Associazione Romana di Entomologia 25(2): 35-43. [in Italian]

Grandi G (1958) Campagna di ricerche dell'Istituto di Entomologia dell'Università di Bologna nel parco Nazionale d'Abruzzo. Sguardo d' insieme ai risultati della campagna. Bollettino dell'Istituto di Entomologia dell'Università di Bologna 23: 133-166. [in Italian]

Kubáň V, Volkovitsh MG, Kalashian MYu, Jendek E (2016) Buprestidae. In: Löbl I, Löbl D (Eds) Catalogue of Palaearctic Coleoptera. Volume 3. Revised and updated edition. Scarabaeoidea, Scirtoidea, Dascilloidea, Buprestoidea and Byrrhoidea. Brill, Leiden Boston, pp. 19-32, 432-574.

Luigioni P (1931) Terzo contributo alla conoscenza della fauna entomologica del Parco Nazionale d'Abruzzo. Atti della Pontifica Academia Romana dei nuovi Lincei 84 Sessione I: $120-180$. [in Italian]

Luigioni P (1933) Quinto contributo alla conoscenza della fauna entomologica del Parco Nazionale d’Abruzzo. Atti della Pontifica Academia Romana dei nuovi Lincei 87 Sessione III: 3-35. [in Italian]

Parenti A (1962) Elenchi di Coleotteri raccolti nel Parco Nazionale d'Abruzzo. Bollettino dell'Associazione Romana di Entomologia 17(1): 1-4. [in Italian]

Parenti A (1964) Elenchi di Coleotteri raccolti nel Parco Nazionale d'Abruzzo. Bollettino dell'Associazione Romana di Entomologia 19(3-4): 34-36. [in Italian]

Parenti A (1966) Contributo alla conoscenza dei Criocephalus italiani (Coleoptera Cerambycidae). Bollettino dell'Associazione Romana di Entomologia 21(1): 1-4. [in Italian]

Pesarini C, Sabbadini A (2007) Ricerche sui Dorcadiini di Grecia. II. Le specie della Grecia centromeridionale e quelle del gruppo di Dorcadion kozanii (Coleoptera Cerambycidae). Atti della Societa Italiana di Scienze Naturali e del Museo Civico di Storia Naturale in Milano 148(1): 35-83. [in Italian]

Raffray A (1914) Notes sur les Psélaphides d'Italie centrale. Annales de la Société Entomologique de France 83: 369-398. [in French]

Saccà G, Rivosecchi L (1958) Ricerche sulla speciazione nelle mosche domestiche V. Lareale di distribuzione delle subspecie di Musca domestica L. (Diptera: Muscidae). Rendiconti dell'Istituto Superiore di Sanità 21: 1149-1189. [in Italian]

Sama G (1988) Fauna d'Italia. Coleoptera Cerambycidae. Ed. Calderini, Bologna 36, 216 pp. [in Italian]

Sama G, Rapuzzi P (2011) Una nuova Checklist dei Cerambycidae d'Italia (Insecta Coleoptera Cerambycidae). Quaderno di Studi e Notizie di Storia Naturale della Romagna 32: 121-164. [in Italian] 
Sakalian V (1994) Studies on Buprestidae (Coleoptera) in the Sandanski - Petric and Goce Delcev valleys - Southwest Bulgaria. III. Zoogeographical characteristic. Acta zoologica bulgarica 47: 35-42.

Sakalian V, Langourov M (2007) Fauna and Zoogeography of Jewel Beetles (Coleoptera: Buprestidae) in Bulgaria. In: Fet V, Popov A (Eds) Biogeography and Ecology of Bulgaria. Monographiae Biologicae, Springer 82: 3, pp. 57-378.

Sakalian V, Migliaccio E, Tassi F, Doychev D, Georgiev G (2020) New and interesting records of jewel and longhorn beetles of Abruzzo, Lazio and Molise National Park, Italy (Coleoptera: Buprestidae and Cerambycidae). Fragmenta entomologica 52(1): 63-66.

Tassi F (1961) Addenda all'Entomofauna del Parco d'Abruzzo e aree limitrofe. Folia Entomologica 9: 3-4. [in Italian]

Tassi F (1962a) Elenchi di Coleotteri raccolti nel Parco Nazionale d'Abruzzo. Bollettino dell'Associazione Romana di Entomologia 17(1): 4-5. [in Italian]

Tassi F (1962b) Su alcuni Buprestidi italiani specialmente delle regioni centro-meridionali.

Bollettino della Società Entomologica Italiana 92(3-4): 53-57. [in Italian]

Tassi F (1963) Alcuni interessanti longicorni del Parco Nazionale del Circeo. Bollettino dell'Associazione Romana di Entomologia 18(2-3): 19-22. [in Italian]

Tassi F (1964a) Un iteressante reperto nel parco nazionale d'Abruzzo: l'Anthaxia godeti Cast. G. Bollettino dell'Associazione Romana di Entomologia 19(1): 10-13. [in Italian]

Tassi F (1964b) Elenchi di Coleotteri raccolti nel Parco Nazionale d'Abruzzo. Bollettino dell'Associazione Romana di Entomologia 19(3-4): 34-36. [in Italian]

Tassi F (1966) Su alcuni Buprestidi Italiani nuovi o particolarmente interessanti. Bollettino della Società Entomologica Italiana 96(1-2): 17-27. [in Italian]

Tassi F (1980) Nuovi reperti della Coleotterofauna fitoxylofaga d’Abruzzo. Folia Entomologica 28: 14-15. [in Italian]

Topalov P (2018) A check list and areography of longhorn beetles (Coleoptera: Cerambycidae) in Vitosha Mountain. Silva balcanica 3: 21-40.

Vigna Taglianti A (1994) Un nuovo Ocys dell'Appennino Abruzzese (Coleoptera Carabidae). Bollettino dell'Associazione Romana di Entomologia 49(3-4): 129-136. [in Italian]

Vigna Taglianti A (1995) Progetto Biodiversità. Liste preliminari degli organismi viventi nel Parco Nazionale d'Abruzzo (Coleoptera: Carabidae) 1: 5-32. [in Italian] 